\title{
Wahrnehmung des Energieausweises in hypothetischen und realen Mietentscheidungen auf dem deutschen Wohnimmobilienmarkt
}

\author{
Bertram I. Steininger · Claudia Nadler $\cdot$ Melanie Franke • \\ Carolin Pommeranz
}

Eingegangen: 2. August 2018 / Angenommen: 2. Dezember 2018 / Online publiziert: 18. Dezember 2018 (C) Der/die Autor(en) 2018

Zusammenfassung Die Immobilienbranche nimmt eine Schlüsselfunktion in der Klimaschutz- und Energiepolitik Deutschlands ein, da durch sie große Einsparungspotenziale bei Energieverbrauch und $\mathrm{CO}_{2}$-Emissionen identifiziert werden. Der Energieausweis stellt ein wichtiges Informationsinstrument dar, um Aufschluss über die energetische Qualität einer Immobilie zu erlangen. In der vorliegenden Studie werden die Wahrnehmung und der Einfluss des Energieausweises anhand sich ergänzender Methoden untersucht: Experteninterviews, Choice-Based Conjoint-Analyse (CBCA) und hedonisches Preismodell. Neben der Analyse hypothetischer Mietentscheidungen bei Wohnimmobilien steht auch der Preiseffekt des Energieausweises in realen Mietentscheidungen im Fokus dieser Untersuchung. Die Ergebnisse zeigen zum einen eine vergleichsweise hohe Bedeutung der energetischen Qualität einer Immobilie bei der indirekten Ermittlung von Präferenzen im Rahmen der CBCA. Zum anderen ergibt sich aufgrund des vorhandenen Bewusstseins bei der realen Mietentscheidung ein positiver Preiseffekt für energetisch effiziente Wohnungen von bis zu 7,1\%. Eine Intensivierung der Preiseffekte nach Anpassung der gesetzlichen Rahmenbedingungen spricht für eine höhere Markttransparenz durch die Einführung der Vorlagepflicht des Energieausweises. Es bestehen jedoch weiterhin Defizite in

Vorherige Versionen dieses Beitrags wurden als Working Paper auf mehreren Plattformen veröffentlicht. Deren Namen wurden der Editorin mitgeteilt.

\footnotetext{
B. I. Steininger $(\bowtie)$

KTH Royal Institute of Technology, School of Architecture and the Built Environment, 100 44 Stockholm, Schweden

E-Mail: bertram.steininger@abe.kth.se

C. Nadler · M. Franke

Lehrstuhl für Betriebswirtschaftslehre, insbes. Betriebliche Finanzwirtschaft, RWTH Aachen University, Templergraben 64, 52056 Aachen, Deutschland

C. Pommeranz

Institut für Real Estate Finance, RWTH Aachen University, Templergraben 64, 52056 Aachen, Deutschland
} 
der Umsetzung, sodass abschließend Empfehlungen für eine Weiterentwicklung des Informationsinstruments gegeben werden.

Schlüsselwörter Energieausweis · Vorlagepflicht · Wohnimmobilien · ConjointAnalyse · Preiseffekt

\title{
Perception and Price Effect of Energy Performance Certificates on the German Residential Market
}

\begin{abstract}
The real estate sector plays a key role in the German climate and energy policy due to its great potential for reducing the energy consumption and $\mathrm{CO}_{2}$ emissions. The Energy Performance Certificate (EPC) represents an important tool to provide information concerning the energy quality of a certain property. The introduction of the obligation to disclose the EPC when residential buildings are offered for sale or rent in 2014 should further strengthen the role of the information tool. This study investigates the perception and influence of the EPC using the following complementary methods: expert interviews, choice-based conjoint analysis and hedonic pricing model. In addition to the analysis of hypothetical rental decisions on residential properties, this study also focuses on the resulting price effect of EPCs. The results show on the one hand a relatively high importance of the energy quality when tenants' preferences are determined indirectly. On the other hand, the price effect for energy-efficient apartments amounts up to $7.1 \%$. The price effect also intensifies after the recast of the legal provisions indicating a higher transparency of the market through the introduction of the disclosure obligation. However, there are still obstacles, which require further development of the information tool in order to exploit the potential of the EPC.
\end{abstract}

Keywords Energy Performance Certificate $\cdot$ Obligation to Disclose $\cdot$ Residential Properties · Conjoint Analysis · Price Effects

\section{Energieeffizienz in der Immobilienbranche}

Bei der Erreichung energie- und klimapolitischer Ziele wird der Immobilienbranche eine Schlüsselfunktion zugesprochen: Auf den Gebäudesektor entfallen global $32 \%$ des Endenergieverbrauchs, $19 \%$ der $\mathrm{CO}_{2}$-Emissionen sowie $51 \%$ des Stromverbrauchs (IPCC 2014). Dementsprechend wird in der Baubranche das höchste Einsparpotenzial von Treibhausgasen im Vergleich zu anderen Sektoren wie der Landwirtschaft, der Industrie oder dem Transport identifiziert (Ürge-Vorsatz und Novikova 2008). In Deutschland werden im Zuge der eingeleiteten Energiewende verschärfte energetische Anforderungen an Immobilien gestellt. Im Fokus der Energiepolitik steht der Gebäudebereich, da der Primärenergieverbrauch des gesamten Gebäudebestands bis zum Jahr 2050 gegenüber 2008 um $80 \%$ reduziert sowie die restlichen $20 \%$ durch erneuerbare Energien abgedeckt werden sollen (Schafhausen 2013). 
Von Interesse sind neben ökologischen auch ökonomische Aspekte. Vor dem Hintergrund steigender Wärme- und Strompreise nimmt die Attraktivität energieeffizienter und somit energiesparender Immobilien zu. In Deutschland ist das Ausgangsniveau beim energiesparenden, ressourcenschonenden und gesundheitsgerechten Planen, Bauen und Betreiben von Immobilien aufgrund des vorhandenen Umweltbewusstseins der Bevölkerung bereits vergleichsweise hoch (Bundesministerium für Umwelt, Naturschutz, Bau und Reaktorsicherheit (BMUB) 2016, 2017; Kaiser et al. 2011; Lützkendorf und Lorenz 2007). Zudem ist der Anteil erneuerbarer Energien am Stromverbrauch zwischen den Jahren 2005 und 2015 von $10 \%$ auf $32 \%$ gestiegen (Statistisches Bundesamt 2017). Dies repräsentiert das gewachsene ökologische Bewusstsein und das steigende Interesse an nachhaltigem Wirtschaften, das auch mithilfe von politischen Maßnahmen wie Subventionen und Energieausweis vorangetrieben worden ist.

Im Jahr 1976 verabschiedete der Deutsche Bundestag das Energieeinsparungsgesetz (EnEG), das den Grundstein für die folgenden gesetzlichen Regelungen zur Umsetzung ökologischer Standards legte. Seit dem Jahr 2001 stellt die darauf aufbauende Energieeinsparverordnung (EnEV) Mindestanforderungen an die energetische Qualität von Gebäuden, um den Energie-, Heizungs- und Warmwasserbedarf zu senken (Ebert et al. 2010). Die EnEV ist nicht nur für Eigentümer von Bedeutung, sondern aufgrund des im Jahr 2008 eingeführten Energieausweises auch für Mieter und Käufer. Dieser soll eine Bewertung des energetischen Zustands einer Immobilie bereits vor der Miet- bzw. Kaufentscheidung anhand von Bedarfs- bzw. Verbrauchszahlen ${ }^{1}$ ermöglichen. Jedoch bestand zunächst keine Ausweispflicht, sodass der Energieausweis lediglich nach Forderung der Einsichtnahme zugänglich zu machen war (EnEV 2007). Mit der letzten Novelle der EnEV, die im Jahr 2014 in Kraft trat, sowie mit den EU-Richtlinien 2010/31/EU und 2012/27/EU wurde nunmehr das Ausweisen der Energieeffizienz eines jeden Gebäudes veranlasst, was für mehr Markttransparenz sorgen soll. Die Übergangsfrist dieser Anordnung endete zum 1. Mai 2015. Vermieter und Verkäufer sind mit wenigen Ausnahmen dazu verpflichtet, die Energieeffizienz ihrer Immobilie klassifizieren zu lassen und in einer Immobilienanzeige auszuweisen bzw. dem Mieter oder Käufer unaufgefordert vorzulegen.

Ziel des Energieausweises ist es, ein einheitliches, kostengünstiges sowie leicht verständliches Instrument bereitzustellen, das über die energetische Qualität eines Gebäudes aufklärt. Zudem soll der Energieausweis zu einem deutschlandweiten, objektiven Vergleich der Energieeffizienz von Gebäuden beitragen. Aus ökonomischer Perspektive kann der Energieausweis durch Preissignale dazu motivieren, die Energieeffizienz in die Miet- bzw. Kaufentscheidung einfließen zu lassen. Ein Nutzenzuwachs durch eine energieeffiziente Immobilie kann sich in unterschiedlicher Weise als monetärer Preiseffekt niederschlagen: Zum einen, indem Energiekosten durch einen niedrigeren Energieverbrauch reduziert werden, zum anderen durch indirekte Effekte wie einen höheren Werterhalt, einen steigenden Wohnkomfort oder eine Art Versicherung gegenüber potenziell steigenden Energiekosten.

\footnotetext{
${ }^{1}$ Der Bedarfsausweis bezieht sich auf den berechneten Energiebedarf des Gebäudes, wohingegen der Verbrauchsausweis auf dem vergangenen gemessenen Energieverbrauch beruht (Weglage 2010).
} 
Generell wird jedoch kritisiert, dass die unterschiedlichen Ausstellungsformen als Bedarfs- sowie Verbrauchsausweis und ihre Implikationen für den Verbraucher oft intransparent und schwer zu vergleichen sind. Der Unterschied zwischen den Energiekennwerten von Bedarfs- und Verbrauchsausweis liegt beispielsweise durchschnittlich bei $25 \%$ (Verbraucherzentrale NRW 2018). Durch die stetigen Novellen der EnEV in den Jahren 2009 sowie 2014 sind zusätzlich die Vergleichswerte zur Einordnung der Endenergiekennwerte so grundlegend verändert worden, dass ältere Ausweise nicht für einen Vergleich geeignet sind. Die stetige Verbesserung der gesetzlichen Grundlage ist zwar von Vorteil im Hinblick auf die Aktualität der Vorgaben, sorgt jedoch für eine begrenzte Vergleichbarkeit der Zertifizierung. Die Wirksamkeit des Energieausweises beruht letztlich sowohl auf dem Vertrauen in die Objektivität des Instruments als auch auf der Bereitschaft, aus energetischen Schwächen des Gebäudes resultierende Konsequenzen abzuleiten. Potenzielle Mieter und Käufer sollten ein Objekt mit besserem Energie-Effizienzstandard trotz eines höheren Preises bevorzugen und Eigentümer in Sanierungs- sowie Modernisierungsmaßnahmen investieren.

\section{Stand der Forschung und Forschungsfragen}

Bisherige Studien beschäftigen sich vorwiegend mit dem Einfluss des Energieausweises auf die Kaufentscheidung einer Immobilie und weisen auf bestehende Barrieren hin. Adjei et al. (2011) zeigen auf, dass innerhalb Europas, u.a. auch in Deutschland, ein hoher Bekanntheitsgrad sowie ein Grundverständnis von Energieausweisen vorhanden sind. Jedoch vertrauen lediglich $40 \%$ der Befragten den enthaltenen Informationen. Eine Studie von Amecke (2012) beschäftigt sich ebenfalls mit der Bedeutung der Energieeffizienz von Immobilien auf dem deutschen Markt. Über $80 \%$ der Befragten geben an, den Energieausweis zu kennen. Rund $78 \%$ nutzen ihn darüber hinaus für die Immobiliensuche. Als Kaufkriterium wird der Energieeffizienz im Vergleich zu anderen Immobilienmerkmalen - z. B. Preis, Lage oder Größe - trotzdem ein geringer Einfluss beigemessen. Weitere Studien zum europäischen Markt liefern ähnliche Ergebnisse (vgl. u.a. Christensen et al. 2014; Murphy 2014; Watts et al. 2011; Zalejska-Jonsson 2013). Zu kritisieren ist u. a., dass der Energieausweis zu selten aktiv vorgelegt und das Potenzial als Informationsinstrument untergraben wird (Amecke 2012). Vor dem Hintergrund der Einführung der verbindlichen Vorlagepflicht im Jahr 2014 ist zu untersuchen, ob sich die Wahrnehmung durch die Marktakteure verändert hat und wie die Bedeutung des Instruments auf lokalen Immobilienmärkten beurteilt wird. Daraus ergibt sich folgende Forschungsfrage für diese Studie:

1. Wie werden der Umgang mit dem Thema Energieeffizienz und die Rolle des Energieausweises als Entscheidungskriterium von unterschiedlichen Marktakteuren (z. B. Interessenvertretung von Mietern sowie Eigentümern) eingeschätzt?

Im Gegensatz zu bisherigen Forschungsarbeiten sollen die Immobilienmerkmale nicht ausschließlich separat (d.h. direkt) abgefragt, sondern eine Immobilie vielmehr als Nutzenbündel verstanden werden, das durch verschiedene Eigenschaften 
gekennzeichnet ist. Vorteile werden in der Vermeidung von ,,information bias“ und „strategic bias“ gesehen, die zu Antwortverzerrungen und Problemen bei der Ableitung absoluter Zahlungsbereitschaften führen (Schober 2015). Die indirekte Ermittlung von Präferenzen hat daher in den letzten Jahren einen Bedeutungszuwachs erfahren und wird mittlerweile sowohl bei energieeffizienz-spezifischen als auch bei immobilien-spezifischen Themen genutzt. Sammer und Wüstenhagen (2006) zeigen beispielsweise, dass bei Waschmaschinen ein Einfluss der Energieeffizienzklasse auf das Konsumentenverhalten vorhanden ist. Auch im Immobilienbereich findet die indirekte Ermittlung Anwendung, indem beispielsweise konventionelle und „Green Buildings“ gegenübergestellt werden oder die Zahlungsbereitschaft für Energieeinsparmaßnahmen erhoben wird (Banfi et al. 2008; Chau et al. 2010; Mansour und Radford 2016). Offen bleibt bisher jedoch, inwiefern der Energieausweis die Wahrnehmung bei der privaten Mietentscheidungsfindung beeinflusst. Das Entscheidungsverhalten wird in hypothetischen Auswahlsituationen untersucht, um sich der folgenden Forschungsfrage zu widmen:

2. Inwiefern beeinflusst die Energieeffizienz einer Wohnimmobilie die (hypothetische) Mietentscheidung?

Damit der Wert des Energieausweises tatsächlich zum Tragen kommt, müsste eine Auswahlsituation mit ausreichend Vergleichsobjekten auf dem realen Immobilienmarkt vorhanden sein. Der Fokus der Forschungsarbeit liegt im Folgenden nicht nur auf hypothetischen Auswahlsituationen, sondern auch auf dem Einfluss in realen Immobilienmärkten. Der sich einstellende Preiseffekt für den Energieausweis kann anhand eines hedonischen Preismodells gemessen werden. Die aktuelle Forschung hinsichtlich energieeffizienter Immobilien konzentriert sich bislang auf freiwillig zertifizierte, gewerbliche Immobilien. Eichholtz et al. (2010) identifizieren in den USA einen Mietpreisaufschlag für zertifizierte Büroimmobilien von bis zu $3 \%$ und für Kaufimmobilien von bis zu 16\%. Ähnliche Ergebnisse liefern beispielsweise Studien zu den Niederlanden (Kok und Jennen 2012). Für den größeren Wohnimmobilienmarkt existieren jedoch trotz der offensichtlichen Relevanz für eine nachhaltige Entwicklung der Gesamtwirtschaft weniger Studien. Gründe können teilweise in einer höheren Fragmentierung der Eigentümer und Nutzer oder einem geringeren Anteil an institutionellen Investitionen im Vergleich zum gewerblichen Immobilienmarkt liegen. Kahn und Kok (2014) zeigen, dass US-amerikanische Hauskäufer bereit sind, einen Preisaufschlag für Immobilien mit einem Energielabel zu zahlen. Fuerst et al. (2015) belegen diese Ergebnisse für Großbritannien und identifizieren einen Aufschlag von $5 \%$ für Immobilien der höheren Effizienzklassen im Vergleich zur mittleren Referenzkategorie. Die vorliegende Studie konzentriert sich auf den Wohnimmobilienmarkt in Nordrhein-Westfalen (NRW), der als repräsentativer deutscher Teilmarkt angesehen werden kann: er beinhaltet sowohl Großstädte und mittelgroße Städte als auch ländlich geprägte Regionen sowie wachsende und schrumpfende Märkte, die durch Zu- bzw. Abwanderung geprägt sind. NRW als bevölkerungsreichstes Bundesland Deutschlands zeichnet sich durch steigende Bevölkerungs- und Haushaltszahlen aus, die zu einer hohen Nachfrage im Wohnimmobilienmarkt, vor allem in Großstädten, führen (LEG Immobilien AG 2018). Die 
Entwicklung ebendieses Teilmarktes ist daher von besonderer Bedeutung, um die folgende Forschungsfrage zu beantworten:

3.a Stellt sich ein Preiseffekt für den Energieausweis bei Neuvermietungen ein und wenn ja, in welchem Verhältnis steht dieser Effekt zu den durchschnittlichen Energiekosten?

Der Wohnungsmarkt in NRW ist durch eine hohe Komplexität, ausgedehnte Ballungsräume, regionales Bevölkerungswachstum sowie durch Marktsegmente mit einem Ungleichgewicht von Angebot und Nachfrage gekennzeichnet (LEG Immobilien AG 2018; Henger et al. 2015). Dies zeigt sich auch im heterogenen Mietpreisniveau, sodass die unterschiedlichen Märkte der Ballungszentren in angespannt und entspannt eingeordnet werden können (Deschermeier et al. 2015). In angespannten Mietmärkten, in denen ein Überhang der Nachfrage vorhanden ist, ist es möglich, dass das Potenzial des Energieausweises nicht ausgeschöpft werden kann. Von Interesse ist, wie sich die Wohnungsmarktsituation auf die Preiseffekte für Energieeffizienzklassen auswirkt. Immobilienmärkte mit einem deutlichen Nachfrageüberhang verleihen den Vermietern mehr Marktmacht, sodass sich höhere Preiseffekte für eine hohe Energieeffizienz einstellen könnten. Eine Abhängigkeit des Preiseffektes von der Wohnungsmarktsituation wurde zudem bereits in der Literatur nachgewiesen (z. B. Hyland et al. 2013).

Mit dem Auslauf der Übergangsfrist für die Vorlagepflicht des Energieausweises im Mai 2015 sollte sich eine erhöhte Markttransparenz eingestellt haben. Potenzielle Mieter müssten nun bezüglich des energetischen Zustands einer Wohnung und bautechnisch benötigter Modernisierungsmaßnahmen vollständig informiert sein. $\mathrm{Zu}$ erwarten wäre eine Erhöhung des Preisaufschlags für energieeffiziente Mietwohnungen sowie eine Verstärkung des Preisabschlages für wenig effiziente Wohnungen. Die Analyse von Preiseffekten wird daher durch die folgende Fragestellung erweitert:

3.b Wie wirken sich a) die Immobilienmarktsituation und b) die Einführung der verbindlichen Vorlagepflicht des Energieausweises im Jahr 2014 auf einen möglichen Preiseffekt aus?

\section{Untersuchungsmethode}

Zur Beantwortung der Forschungsfragen werden drei verschiedene Methoden verwendet: eine Expertenbefragung und eine experimentelle Analyse - exemplarisch auf den Aachener Immobilienmarkt zugeschnitten - sowie eine empirische Analyse des gesamten Immobilienmarktes in NRW.

\subsection{Experteninterviews}

Zunächst sind sechs Experteninterviews durchgeführt worden, um die Wahrnehmung des Energieausweises und dessen Informationsgehalt grundlegend einschätzen zu können. Zur Beantwortung der ersten Forschungsfrage sind Akteure aus den Berei- 
chen der Energieberatung, der Interessenvertretung von Mietern und Eigentümern sowie des Verbraucherschutzes im Rahmen individueller, ca. einstündiger Interviews befragt worden. Die Liste der Interviewpartner kann beim Autorenteam angefragt werden. Da sich lokale Immobilienmärkte in NRW stark unterscheiden können, liegt der Fokus beispielhaft auf dem Aachener Immobilienmarkt, der auch für die spätere experimentelle Analyse genutzt wird. Die Städteregion Aachen erweist sich als geeigneter Teilmarkt, da sie zu den zwölf wichtigsten Standorten in NRW gehört (LEG Immobilien AG 2018) und eine Bautätigkeit aufweist, die in etwa dem Baubedarf entspricht (Henger et al. 2015). Dies gewährleistet, dass - im Gegensatz zu angespannten Märkten - für potenzielle Interessenten tatsächlich eine Auswahlsituation auf dem lokalen Immobilienmarkt vorhanden ist.

\subsection{Experimentelle Analyse}

Die Methode der auswahlbasierten Conjoint-Analyse, der sogenannten ChoiceBased Conjoint-Analyse (CBCA), bietet die Möglichkeit, den Einfluss der Energieeffizienz auf die Objektwahl bei der Mietentscheidung abzubilden. Die indirekte Ermittlung von Präferenzen mittels der CBCA wird als vorteilhaft angesehen, weil fraglich ist, ob Probanden ihre Präferenzen eindeutig kommunizieren können (Green und Srinivasan 1990; Sattler und Hensel-Börner 2001). Ein weiterer Vorteil der Methode wird in der Darstellung einer möglichst realitätsnahen Entscheidungssituation gesehen, indem ein „Produkt“ als Ganzes bewertet wird im Gegensatz zu isolierten Einschätzungen einzelner Produkteigenschaften (McCullough 2002, Schober 2015). Der Energieverbrauch kann dabei als eine Produkteigenschaft einer Immobilie interpretiert werden. Probanden bewerten die Wichtigkeit der Energieeffizienz bei einer Mietentscheidung somit nicht direkt und isoliert, sondern müssen Auswahlentscheidungen zwischen verschiedenen Wohnungen treffen. Diese Wohnungen unterscheiden sich durch einen bestimmten Energieverbrauch, aber auch durch weitere Charakteristika - z. B. Lage und Preis.

Für die CBCA sind sechs Immobilienmerkmale als Produkteigenschaften ausgewählt worden, denen der mitunter größte Einfluss auf die Kauf- oder Mietentscheidung zugeschrieben wird (Brounen et al. 2013; Fuerst et al. 2015; Kok und Jennen 2012; Amecke 2012). Die Konzentration auf sechs Merkmale ist darauf zurückzuführen, dass die Probanden nicht überfordert werden sollten (,,information overload problem“) (Green und Srinivasan 1978; Orme 2002). Um ein ausgeglichenes Design zu gewährleisten, weisen alle Immobilienmerkmale, die sogenannten Attribute, die gleiche Anzahl an Leveln mit jeweils drei Ausprägungen auf (McCullough 2002). Die Ausprägungen der Attribute basieren sowohl auf dem Aachener Mietspiegel 2016 als auch auf einem Datensatz von ImmobilienScout24, der Aachener Immobilien aus den Jahren 2011 bis 2016 beinhaltet. Damit die Immobilien vergleichbar bleiben, konzentriert sich die Conjoint-Analyse auf sogenannte Normalwohnungen mit einer Größe von 45 bis $100 \mathrm{~m}^{2}$, für die ein Quadratmeterpreis von 3,20 bis $10,80 €$ gezahlt wird (Mieterschutzverein für Aachen und Umgegend e. V. et al. 2016). Auf Basis dieser Einschränkungen sind 3268 Aachener Immobilien herangezogen worden, um sinnvolle Ausprägungen der jeweiligen Eigenschaften festzulegen. Hierzu sind der Mittelwert sowie das 10. und 90. Perzentil ausgewählt worden. 


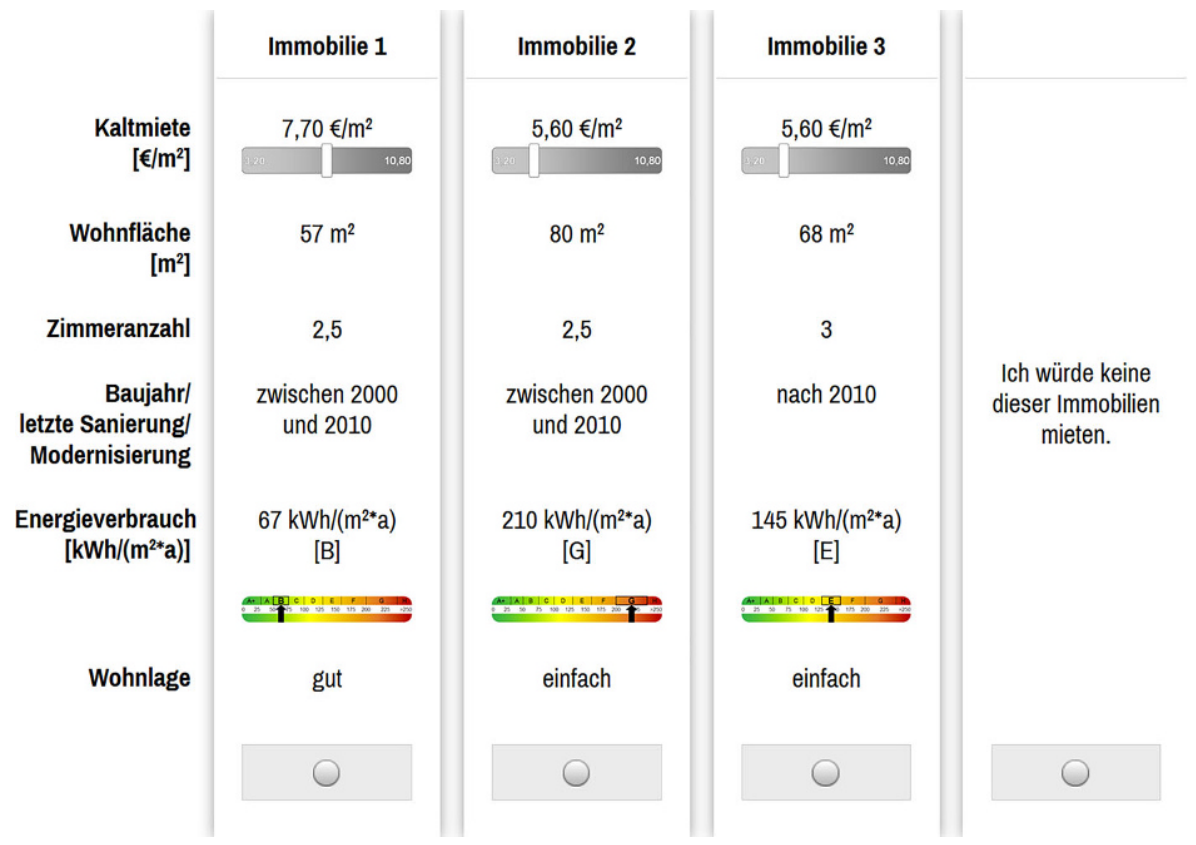

Abb. 1 Beispielhafte Auswahlsituation der CBCA

Alle Eigenschaften wurden zu Beginn der Umfrage erläutert, um sicherzustellen, dass ein hinreichendes Verständnis seitens der Teilnehmer vorliegt. Jede der zwölf fiktiven Auswahlsituationen beinhaltete neben drei Immobilien, die sich hinsichtlich der sechs Merkmale unterschieden, auch die Möglichkeit, keine der angebotenen Immobilien zu wählen (vgl. Abb. 1).

Aus den getroffenen Auswahlentscheidungen können Teilnutzen für die einzelnen Produkteigenschaften abgeleitet werden, um wiederum Rückschlüsse auf deren relative Wichtigkeit bei einer Mietentscheidung zu ziehen. Die Analyse erfolgt anhand einer Hierarchical-Bayes-Schätzung mittels Sawtooth Software. Die Methode ermöglicht es, die Nutzenfunktion der Probanden auf einem individuellen Level auf der Basis weniger Auswahlentscheidungen zu schätzen, was sowohl die Validität als auch die Vorhersagequalität der Daten verbessert (Orme 2000). Hierdurch wird die zweite Forschungsfrage hinsichtlich des Einflusses der Energieeffizienz bei Mietentscheidungen behandelt.

Nach Abschluss der CBCA sind die Teilnehmer zudem direkt zum Energieausweis befragt worden, um dessen Informationswert sowie Nützlichkeit bestimmen zu können. Hierzu mussten die teilnehmenden Personen Bewertungen auf einer 5-stufigen Skala von ,stimme überhaupt nicht zu“ (=1) bis ,stimme voll zu“ (=5) vornehmen. 


\subsection{Empirische Analyse}

Preiseffekte für die im Energieausweis ausgewiesenen Effizienzklassen werden implizit anhand einer hedonischen Preisregression nach Rosen (1974) bestimmt. Mithilfe des hedonischen Modells lassen sich Immobilien als heterogene Güter in ihre jeweiligen Eigenschaften - Größe, Zimmeranzahl, Lage etc. - zerlegen, um schließlich anhand einer Regression den Einfluss jeder einzelnen Eigenschaft auf den Immobilienpreis auszuweisen. Dadurch ist es möglich, auch den Preis für nicht-marktgehandelte Eigenschaften zu messen - z. B. die Nähe zu Grünanlagen (Conway et al. 2010).

Für die Analyse werden Daten von ImmobilienScout24, dem führenden Onlineportal für Immobilienanzeigen in Deutschland, genutzt, die sowohl den Angebotsmietpreis ${ }^{2}$ beinhalten als auch strukturelle Merkmale wie Größe, Ausstattung und Energieeffizienzkennwert (Meulen et al. 2014). Der Datensatz wurde mit sozioökonomischen Daten von GfK Geomarketing sowie mit Daten zu relativen Entfernungen zu Infrastruktureinrichtungen und zentralen Punkten aus OpenStreetMap angereichert. Mit 400.212 vollständig erfassten Mietwohnungen über den Zeitraum von $2011^{3}$ bis 2016 kann der Immobilienmarkt für Neuvermietungen in NRW abgebildet werden. ${ }^{4}$ Wenn Anzeigen innerhalb von 24 Monaten aufgrund von Marketingmaßnahmen oder Nichtvermietung mehrfach im Datensatz vorhanden sind, wird nur die letzte Beobachtung verwendet. Die Verweildauer auf dem Portal wird zusätzlich in das Modell integriert. Hierdurch kann eine etwaige Verzerrung kontrolliert werden, die dadurch entsteht, dass eine Abweichung vom antizipierten Transaktionspreis zu einer Erhöhung der Angebotszeit führt. Darüber hinaus erfolgt die Interpretation der Ergebnisse im Verhältnis zu den durchschnittlichen Energiekosten. Die Methode der hedonischen Preisregression wird für die Beantwortung der dritten und vierten Forschungsfrage verwendet.

Im Anschluss gilt es zu analysieren, ob die Preiseffekte von der Immobilienmarktsituation und der Einführung der verbindlichen Vorlagepflicht abhängen. Die Aufteilung in die drei Marktsegmente ,,angespannt“, „,normal“ und „entspannt“ erfolgt auf der Ebene der Postleitzahlgebiete und basiert auf der durchschnittlichen, jährlichen Mietpreisänderung in dem Jahr des Immobilieninserates. Als entspannt gelten dabei Werte unterhalb des dritten Dezils, als angespannt Werte oberhalb des siebten Dezils und als normal die Werte im Zwischenbereich. Ein Interaktionseffekt

\footnotetext{
2 In der vorliegenden Studie werden Angebotspreise für die Analyse genutzt. Der Angebotspreis kann vom Transaktionspreis abweichen, da der tatsächlich gezahlte Preis das Ergebnis eines Verhandlungsprozesses ist (Glück 1997). Henger und Voigtländer (2014) zeigen, dass Angebotsdaten die Preisentwicklung in Deutschland adäquat wiedergeben können.

3 Auch wenn der uns zur Verfügung stehende Datensatz bereits im Jahr 2007 beginnt, haben wir unseren Zeitraum auf nach 2010 begrenzt, da z. B. für das Jahr 2007 nur 0,4\% der rund 300 Beobachtungen einen Energiekennwert aufweisen. Das Jahr 2011 hat erstmals über 10.000 Beobachtungen von denen wiederum $11 \%$ einen Energiekennwert besitzen; dahingegen hat das Jahr 2016 rund 110.000 Beobachtungen wobei $76 \%$ einen Energiekennwert aufweisen.

${ }^{4}$ Der Wohnungsbestand in NRW belief sich im Jahr 2017 auf insgesamt 8.972.774 Wohnungen (Information und Technik Nordrhein-Westfalen, Geschäftsbereich Statistik 2018). Die durchschnittliche Mietdauer in Deutschland beträgt 7,8 Jahre (Landtag Nordrhein-Westfalen 2016).
} 
aus Energieeffizienzklasse und Zeiteffekt (die Immobilie wurde vor bzw. nach Mai 2015 angeboten) bildet die Veränderungen der Preiseffekte durch die Vorlagepflicht ab.

\section{Ergebnisse und Diskussion}

Im Folgenden werden die Ergebnisse der Analysen im Hinblick auf die vier Forschungsfragen vorgestellt und diskutiert.

\subsection{Experteninterviews}

Aus den Experteninterviews resultieren die folgenden drei zentralen Ergebnisse:

1. Soll der Energieausweis als wirksames Instrument zur Verbesserung der Energieeffizienz des Gebäudebestands beitragen, ist es von essenzieller Bedeutung, dass die potenziellen Mieter und Käufer dessen Inhalte verstehen. Unter den befragten Experten besteht jedoch die übereinstimmende Meinung, dass der Energieausweis nicht verständlich gestaltet ist. Dabei wird vor allem das fehlende Wissen über die Bedeutung der Begrifflichkeiten und Kennwerte sowie über die Vorlagepflicht kritisiert.

2. Das mangelnde Verständnis ist zum Teil auf die defizitäre Umsetzung des Energieausweises zurückzuführen. Als problematisch anzusehen ist das duale System des Bedarfs- und Verbrauchsausweises, das die Vergleichbarkeit der enthaltenen Informationen erschwert. Den Energieausweis zu vereinheitlichen und die Nutzer stärker zu sensibilisieren, könnte zu einer Verbesserung des Verständnisses beitragen. Auch die Einführung eines zusätzlichen Kennwertes, um die Heizkosten konkret abschätzen zu können, wird überwiegend als sinnvoll bewertet, wenngleich eine Umsetzung laut Experten in der Praxis Schwierigkeiten mit sich bringen würde. Problematisch sind beispielsweise der Einfluss des Nutzerverhaltens sowie des dualen Systems des Ausweises, die sich auf den monetären Heizkostenkennwert auswirken könnten. Ein weiterer Kritikpunkt besteht in der mangelnden Kontrolle der Vorzeigepflicht, obwohl ein nicht vorhandener Energieausweis eine Ordnungswidrigkeit darstellt und mit einem Bußgeld belegt werden kann.

3. Die erhöhte Zahlungsbereitschaft eines Mieters oder Käufers für eine energieeffiziente Immobilie kann generell aus unterschiedlichen Motiven resultieren - z. B. aus ökologischen Gründen oder zwecks einer verbesserten Wohnqualität. Unter den Experten besteht jedoch Einigkeit darüber, dass für die überwiegende Mehrheit der Marktteilnehmer wirtschaftliche Beweggründe im Vordergrund stehen. Dementsprechend müssen Kosten und Einsparungen aus einer erhöhten Energieeffizienz in einem angemessenen Verhältnis stehen. Den befragten Experten zufolge ist dies jedoch selten der Fall, da die hohen Bau- und Modernisierungskosten oftmals nur zu geringen Einsparungen führen.

Im Rahmen der Experteninterviews wurde deutlich, dass der Energieausweis weiterhin kritisch gesehen wird. Auch mit der Novelle der EnEV im Jahr 2014 kann den bestehenden Defiziten bislang nicht hinreichend entgegengewirkt werden. Die Rolle 
als Entscheidungskriterium wird generell als vergleichsweise gering eingeschätzt. Vor dem Hintergrund steigender Energiekosten besteht jedoch die Vermutung, dass der Energieausweis zumindest aus Mietersicht in Zukunft an Gewicht gewinnen wird. Dies liegt darin begründet, dass Rückschlüsse auf die späteren Nebenkosten möglich sind und Mietern i.d.R. ein Gesamtbudget zur Verfügung steht, das sämtliche Kosten - Kaltmiete, Neben- und Heizkosten - decken muss. Aus Expertensicht sollte der Energieausweis angepasst und verbessert werden, um beispielsweise eine stärkere Verknüpfung mit den resultierenden Kosteneinsparungen zur Verfügung zu stellen.

\subsection{Experimentelle Analyse}

Die Online-Studie zur Bedeutung der Energieeffizienz bei Mietentscheidungen wurde im Mai 2017 auf Basis eines Probandenpools der RWTH Aachen University durchgeführt. Insgesamt haben 102 Personen an der Umfrage teilgenommen. Dabei handelt es sich um je 51 Männer und Frauen mit einem Durchschnittsalter von 30 Jahren. Die relativ junge Altersstruktur steht damit in Verbindung, dass sich junge Menschen besonders häufig in einer Mietsituation befinden.

Die Teilnutzen der jeweiligen Merkmalsausprägungen und die Wichtigkeit der entsprechenden Eigenschaft zur Nutzenbeurteilung der Immobilie sind Tab. 1 zu entnehmen. Die Ergebnisse lassen sich wie folgt interpretieren: Der jeweils „schlechtesten“ Ausprägung der Eigenschaften - z. B. hoher Preis, schlechte Lage etc. - wird durchgehend der geringste Teilnutzen beigemessen. Die aus den Teilnutzen bestimmten relativen Wichtigkeiten machen deutlich, dass sich zwei Gruppen unterscheiden lassen: zum einen Merkmale mit jeweils ca. $25 \%$ relativer Wichtigkeit, zum anderen Merkmale mit jeweils unter $10 \%$. Wie zu erwarten war, wird der Kaltmiete und der Wohnlage ein hoher Einfluss beigemessen. Überraschend ist hingegen, dass auch dem Energieausweis bzw. der Energieeffizienzklasse eine relative Wichtigkeit von $25 \%$ zugewiesen wird.

In Tab. 2 sind die Ergebnisse der direkten Befragung der Teilnehmer dargestellt. Diese beinhalten sowohl die Einstellung zum Energieausweis als auch die Beurteilung der Energieeffizienz im Allgemeinen. Auffällig ist, dass sowohl die Energieeffizienzklasse als auch ein zusätzlicher Kennwert zur Abschätzung der Heizkosten, der zurzeit noch nicht enthalten ist, als sinnvoll erachtet werden. Demzufolge ist es auch aus Sicht der Befragten wichtig, dass die Informationen leicht verständlich und nachvollziehbar sind, damit eine Beurteilung auf Grundlage einiger weniger Werte möglich ist. Ebenso sollte ein zusätzlicher Kennwert für die Heizkosten verstärkt diskutiert werden, da die Kernkompetenz des Energieausweises im Bereich der Kosten zu sehen ist. Der Energieeffizienz wird dementsprechend die höchste Bedeutung im Hinblick auf die Nebenkosten für Heizung und Warmwasser zugeordnet. Rund $58 \%$ der Teilnehmer stimmten der Aussage voll zu (=5), dass die Energieeffizienz einer Wohnimmobilie aufgrund der Nebenkosten wichtig ist. Hinsichtlich des Umweltschutzes gaben mit $24 \%$ deutlich weniger Probanden ihre volle Zustimmung an. 
Tab. 1 Teilnutzen und relative Wichtigkeiten der Immobilienmerkmale $(N=102)$

\begin{tabular}{|c|c|c|c|}
\hline Eigenschaft & Ausprägung & Teilnutzen & $\begin{array}{l}\text { Relative Wichtig- } \\
\text { keit (in \%) }\end{array}$ \\
\hline \multirow[t]{3}{*}{ Kaltmiete $\left[€ / \mathrm{m}^{2}\right]$} & 9,40 & $-84,4$ & \multirow[t]{3}{*}{26,9} \\
\hline & 7,70 & 13,5 & \\
\hline & 5,60 & 71,0 & \\
\hline \multirow[t]{3}{*}{ Wohnfläche $^{\mathrm{a}}\left[\mathrm{m}^{2}\right]$} & 57 & $-21,5$ & \multirow[t]{3}{*}{9,6} \\
\hline & 68 & 12,4 & \\
\hline & 80 & 9,2 & \\
\hline \multirow[t]{3}{*}{ Zimmeranzahl } & 2 & $-10,1$ & \multirow[t]{3}{*}{5,2} \\
\hline & 2,5 & $-1,0$ & \\
\hline & 3 & 11,1 & \\
\hline \multirow{3}{*}{$\begin{array}{l}\text { Zustand (Baujahr/letzte } \\
\text { Sanierung/Modernisierung) }\end{array}$} & Vor 2000 & $-18,6$ & \multirow[t]{3}{*}{7,3} \\
\hline & $2000-2010$ & 2,6 & \\
\hline & Nach 2010 & 16,0 & \\
\hline \multirow{3}{*}{$\begin{array}{l}\text { Energieverbrauch } \\
\left(\mathrm{kWh} /\left(\mathrm{m}^{2} * \mathrm{a}\right)\right. \\
\text { Energieeffizienzklasse })\end{array}$} & $210(\mathrm{G})$ & $-79,5$ & \multirow[t]{3}{*}{25,0} \\
\hline & $145(\mathrm{E})$ & 15,4 & \\
\hline & $67(B)$ & 64,2 & \\
\hline \multirow[t]{3}{*}{ Wohnlage } & Einfach & $-84,38$ & \multirow[t]{3}{*}{26,0} \\
\hline & Mittel & 15,1 & \\
\hline & Gut & 69,3 & \\
\hline
\end{tabular}

${ }^{a}$ Der relativ hohe Teilnutzen für die Wohnfläche von $68 \mathrm{~m}^{2}$ ist auf die hohe Nachfrage dieser WohnungsgröBe zurückzuführen. Die meistgesuchte Wohnung in Deutschland hat durchschnittlich folgende Merkmale: $66 \mathrm{~m}^{2}$, 2,5 Zimmer, $446 €$ Kaltmiete, Basis: ImmobilienScout24-Daten, 40.000 Mietwohnungsanzeigen von Juli 2017 bis Juni 2018 (Nier 2018).

Die Energieeffizienz einer Immobilie findet im Rahmen der analysierten hypothetischen Auswahlentscheidungen eine stärkere Berücksichtigung, als sich anhand der bisherigen Forschungsarbeiten sowie der Experteninterviews vermuten ließ. Mit einer relativen Wichtigkeit von $25 \%$ gehört das Kriterium des Energieverbrauchs zu der Gruppe der wichtigen Merkmale, die die Auswahl entscheidend beeinflussen. Die bisherige Unterschätzung des Einflusses des Energieausweises könnte auf die zurzeit in den meisten Studien vorherrschende isolierte und direkte Beurteilung von Immobilieneigenschaften zurückzuführen sein. Durch die Choice-Based Conjoint-Analyse kann hingegen die unterbewusste Berücksichtigung deutlich gemacht werden, die zum Ausdruck kommt, wenn verschiedene Merkmale gegeneinander abgewogen werden. Somit beeinflusst der Energieausweis nachweislich das Verhalten in hypothetischen Auswahlsituationen. Dabei bietet das Instrument nicht nur Informationen über den energetischen Zustand einer Immobilie, sondern wird der Befragung der Teilnehmer zufolge auch in einem direkten Zusammenhang mit den Nebenkosten einer Wohnung gesehen. 
Tab. 2 Beurteilung des Energieausweises sowie der Bedeutung der Energieeffizienz

\begin{tabular}{lccc}
\hline & Mittelwert & StA & $N$ \\
\hline Die folgenden Informationen des Energieausweises erachte ich als nützlich: & & \\
Energiekennwert $\left(\mathrm{kWh} /\left(\mathrm{m}^{2} * \mathrm{a}\right)\right)$ & 3,4 & 1,09 & 97 \\
Energieeffizienzklasse $\left[\mathrm{A}^{+}\right.$bis H] & 4,0 & 1,08 & 99 \\
Zusätzlicher Kennwert zur Abschätzung der Heizkosten & 4,0 & 0,98 & 96 \\
Die Energieeffizienz einer Wohnimmobilie ist für mich wichtig aufgrund von & & 1,08 \\
Nebenkosten (Heizung und Warmwasser) & 4,3 & 0,92 & 97 \\
Wohnqualität & 3,4 & 1,29 & 101 \\
Umweltschutz & 3,4 & & \\
\hline
\end{tabular}

Die Erhebung erfolgte auf einer Skala von 1 ,stimme überhaupt nicht zu“ bis 5 ,stimme voll zu“ StA Standardabweichung, $N$ Anzahl der Beobachtungen

\subsection{Empirische Analyse}

\subsubsection{Preiseffekt für den Energieausweis}

Die deskriptive Auswertung des Mietwohnungsdatensatzes in NRW zeigt, dass über den gesamten Zeitraum der bevorzugte Energieausweistyp mit $72 \%$ der Verbrauchsausweis ist; dieser weist auch geringere Ausstellungskosten auf. Die Quote fiel jedoch seit dem Jahr 2011 mit 82\% kontinuierlich auf $68 \%$ im Jahr 2016. Abb. 2 zufolge sind Immobilien im hocheffizienten Bereich (Effizienzklasse $\mathrm{A}^{+}$, A oder B) mit zusammen $7 \%$ aller Mietwohnungen seltener vertreten, während die Kategorien D, E und F mit einem Anteil von jeweils mindestens $20 \%$ am häufigsten

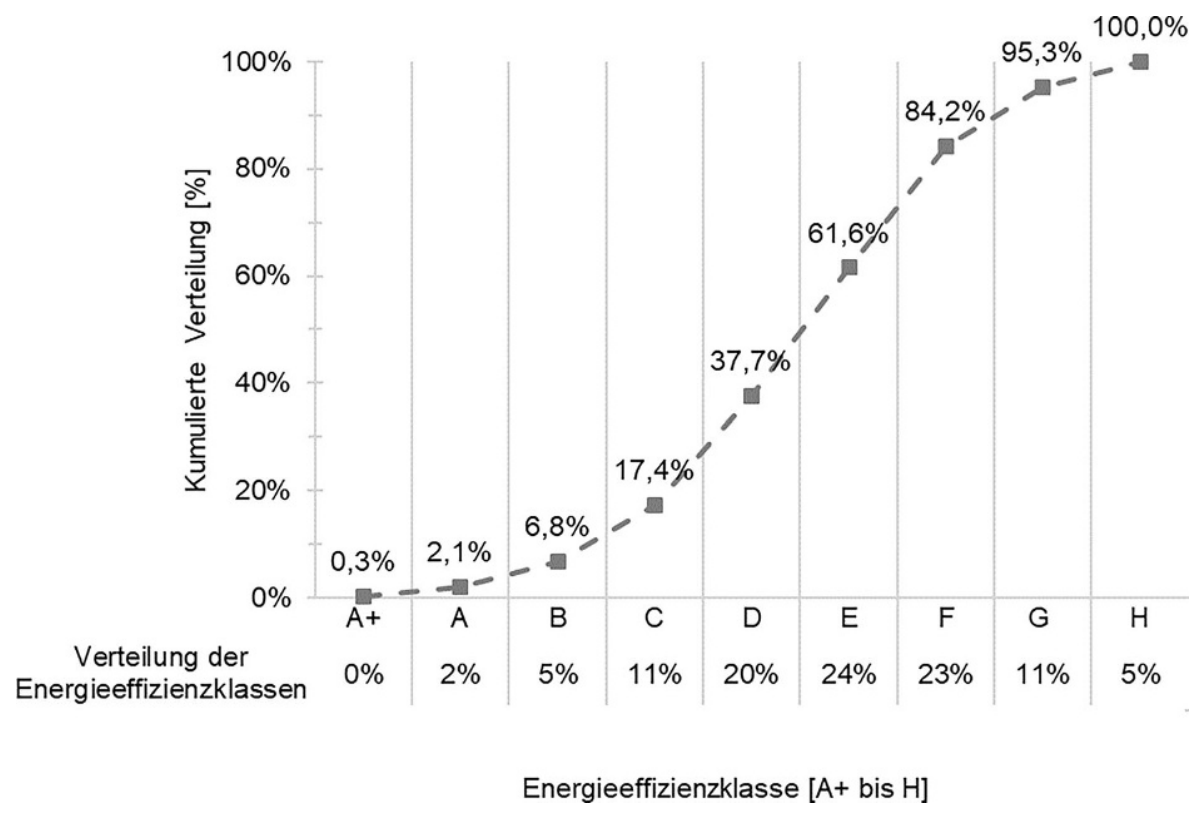

Abb. 2 Verteilung der Energieeffizienzklassen 


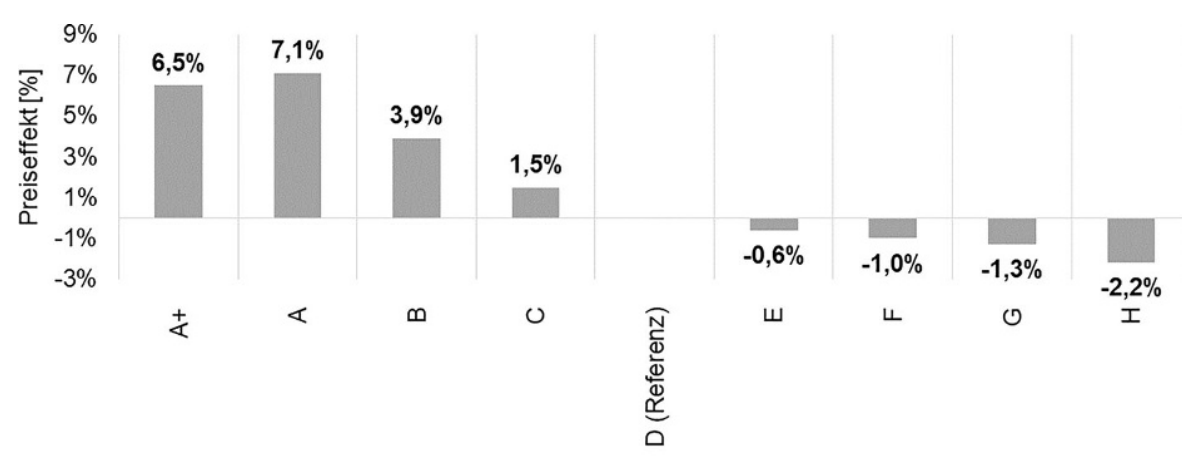

Energieeffizienzklasse $[\mathrm{A}+$ bis $\mathrm{H}]$

Abb. 3 Generelle Auswirkung der Energieeffizienzklasse auf den Mietpreis von Wohnungen in NRW. Die fett dargestellten Werte weisen eine statistische Signifikanz auf dem 0,1\%-Niveau auf

auftreten. Die unteren Energieeffizienzklassen G und H weisen mit zusammen 16\% wiederum einen geringeren Anteil auf.

In Abb. 3 sind die Ergebnisse der hedonischen Preisregression dargestellt. Für energetisch effiziente $\left(\mathrm{A}^{+}\right.$bis $\mathrm{C}$ ) Mietwohnungen stellen sich Preisaufschläge von 1,5 bis $7,1 \%$ ein. Für energetisch wenig effiziente $(\mathrm{E}$ bis $\mathrm{H})$ Mietwohnungen sind hingegen geringe Preisabschläge von $-0,6$ bis $-2,2 \%$ nachweisbar. Der Preiseffekt für eine Wohnung mit einer A-Bewertung übersteigt zudem den für eine $\mathrm{A}^{+}$-Wohnung. Die Ergebnisse sind für alle Effizienzklassen auf dem 1\%-Niveau statistisch signifikant.

Zudem werden die ermittelten Preisdifferenzen den durchschnittlich eingesparten Energiekosten je Effizienzklasse gegenübergestellt (vgl. Tab. 3). Die Energiekosten berechnen sich aus Preisdaten von Verivox unter Berücksichtigung der Merkmale aus dem ursprünglichen Datensatz - z. B. Art der Wärmeerzeugung und Energieverbrauch. Die Werte sind auf Grundlage der getroffenen Annahmen zu interpretieren und sollen lediglich eine Tendenz vorgeben. ${ }^{5}$ Für weitergehende Aussagen bedarf es ggf. einer Weiterentwicklung der hier zu Grunde gelegten Methode. Folgende Angaben gelten jeweils in Bezug auf die Referenzkategorie D: Für Mietwohnungen mit den Energieeffizienzklassen $\mathrm{A}^{+}$bis $\mathrm{C}$ ergibt sich für $\mathrm{A}^{+}$und $\mathrm{C}$ ein positiver Saldo aus zusätzlicher Mietbelastung und Energiekosteneinsparung pro Jahr (im Vergleich zur Referenzkategorie D).

Die positiven Preiseffekte für einen Energieausweis im Bereich $\mathrm{A}^{+}$und $\mathrm{C}$ deuten darauf hin, dass der energetische Zustand einer Immobilie bei Neuvermietungen berücksichtigt wird. Mieter sind dementsprechend bereit, einen Preisaufschlag für eine höhere Energieeffizienzklasse zu zahlen. Die Preisabschläge für die Energieeffizienzklassen $\mathrm{E}$ bis $\mathrm{H}$ weisen jedoch mit $-0,6 \%$ bis $-2,2 \%$ eine ähnliche Höhe

\footnotetext{
5 Die Daten der Energiekosten beziehen sich auf einen Einzelabruf im November 2016 auf Postleitzahlniveau. Da zu dieser Zeit besondere Angebote zu Wechselkonditionen seitens der Anbieter geschaltet wurden, sind die Werte evtl. nicht repräsentativ für das gesamte Jahr sowie für Bestandsverträge. Sofern nicht anders hinterlegt, wird zudem davon ausgegangen, dass Heizkosten nicht in den Nebenkosten innerhalb der Immobilienanzeige von ImmobilienScout enthalten sind.
} 
Tab. 3 Gegenüberstellung des jährlichen Mietpreiseffekts und der jährlich eingesparten Energiekosten

\begin{tabular}{llll}
\hline Energieeffizienzklasse & $\begin{array}{l}\text { Durchschnittlicher Preiseffekt } \\
\text { pro Jahr }[€] \\
\text { (durchschnittlicher Mietpreis: } \\
5984 € / \text { a) }\end{array}$ & $\begin{array}{l}\text { Eingesparte Energiekos- } \\
\text { ten pro Jahr [€] }\end{array}$ & $\begin{array}{l}\text { Saldo } \\
{[€]}\end{array}$ \\
\hline $\mathrm{A}^{+}$ & -389 & +469 & +80 \\
$\mathrm{~A}$ & -425 & +273 & -152 \\
$\mathrm{~B}$ & -233 & +204 & -29 \\
$\mathrm{C}$ & -90 & +109 & +19 \\
$\mathrm{D}$ & Referenzkategorie & & \\
$\mathrm{E}$ & +36 & -128 & -92 \\
$\mathrm{~F}$ & +60 & -287 & -227 \\
$\mathrm{G}$ & +78 & -487 & -410 \\
$\mathrm{H}$ & +132 & -888 & -757 \\
\hline
\end{tabular}

untereinander auf. Mieter sind zwischen der genauen Ausprägung der niedrigeffizienten Energieeffizienzklasse nahezu indifferent, insbesondere dann, wenn sie keine Sensibilität gegenüber dem Wert des Energieausweises aufweisen. Aus der Gegenüberstellung der veränderten Miete mit den eingesparten Kosten durch eine erhöhte Energieeffizienz ergibt sich, dass es für Mieter aus rein monetären Gründen vorteilhaft ist, sich für eine energieeffiziente Wohnung $\left(\mathrm{A}^{+}\right.$und $\left.\mathrm{C}\right) \mathrm{zu}$ entscheiden. Für wenig effiziente Mietwohnungen stellt sich jedoch ein negativer Saldo im Vergleich zur Referenzkategorie D ein, sodass nicht von einem kompensierenden Mietpreisabschlag für die höheren Energiekosten ausgegangen werden kann. Beispielsweise ergibt sich für die Energieeffizienzklasse $\mathrm{H}$ ein negativer Saldo in Höhe von 1,5 durchschnittlichen Monatsmieten (499€).

\subsubsection{Immobilienmarksituation und Vorlagepflicht}

Die Ergebnisse der Analyse unter Einbezug der Angebots-/Nachfragesituation auf dem Immobilienmarkt sind Tab. 4 zu entnehmen. Die Mietpreiseffekte sind meistens auf dem $0,1 \%$-Niveau statistisch signifikant. In angespannten Wohnungsmärkten fallen die Preiseffekte für überdurchschnittliche Effizienzklassen $\left(\mathrm{A}^{+}\right.$bis $\left.\mathrm{C}\right)$ leicht höher aus als in entspannten Marktsegmenten. Lediglich für die Effizienzklasse A ist der Preiseffekt im entspannten Marktsegment mit 8,3\% höher als im angespannten Wohnungsmarkt mit 6,5\%. Die Preisabschläge für wenig effiziente Immobilien sind hingegen nicht von der Immobilienmarktsituation abhängig. Die Abschläge liegen über die verschiedenen Marktsegmente und Energieklassen hinweg zwischen - $0,1 \%$ und $-2,8 \%$.

Die Einführung der Vorlagepflicht führt zu einer leichten Intensivierung der Preiseffekte (vgl. Abb. 4). Der Veränderungseffekt nach Mai 2015 ist für die Effizienzklassen $\mathrm{A}^{+}, \mathrm{C}, \mathrm{E}, \mathrm{F}$ und $\mathrm{G}$ jedoch statistisch nicht auf dem $5 \%$-Niveau signifikant. Alle weiteren Ergebnisse sind mindestens auf dem 0,1\%-Niveau signifikant. Für hocheffiziente Immobilien (Effizienzklasse $\mathrm{A}^{+}$) steigt der Preisaufschlag auf 5,8\%. Der Preisaufschlag für Energieeffizienzklasse A sinkt auf 6,3\% und gleicht sich folglich der Klasse $\mathrm{A}^{+}$an. Die Preisabschläge für weniger effiziente Wohnungen 
Tab. 4 Mietpreiseffekt für Energieeffizienzklassen in Abhängigkeit der Wohnungsmarktsituation

\begin{tabular}{llll}
\hline Energieeffizienzklasse & $\begin{array}{l}\text { Entspannter Wohnungs- } \\
\text { markt (in \%) } \\
\text { Mietpreisänderung } \\
\text { pro Jahr durchschnitt- } \\
\text { lich kleiner als }-1,4 \%\end{array}$ & $\begin{array}{l}\text { Normaler Wohnungs- } \\
\text { markt (in \%) } \\
\text { Mietpreisänderung } \\
\text { pro Jahr durchschnitt- } \\
\text { lich }-1,4 \text { bis 4,7\% }\end{array}$ & $\begin{array}{l}\text { Angespannter Woh- } \\
\text { nungsmarkt (in } \%) \\
\text { Mietpreisänderung } \\
\text { pro Jahr durchschnitt- } \\
\text { lich größer als 4,7\% }\end{array}$ \\
\hline $\mathrm{A}^{+}$ & $6,4 * * *$ & $6,1 * * *$ & $6,6 * * *$ \\
$\mathrm{~A}$ & $8,3 * * *$ & $6,7 * * *$ & $6,5 * * *$ \\
$\mathrm{~B}$ & $3,6 * * *$ & $3,1 * * *$ & $4,9 * * *$ \\
$\mathrm{C}$ & $1,5 * * *$ & $0,9 * * *$ & $2,1 * * *$ \\
$\mathrm{D}$ & Referenzkategorie & & $-0,5 * * *$ \\
$\mathrm{E}$ & $-0,1$ & $-1,1 * * *$ & $-1,2 * * *$ \\
$\mathrm{~F}$ & $-0,4 * *$ & $-1,4 * * *$ & $-1,0 * * *$ \\
$\mathrm{G}$ & $-1,0 * * *$ & $-1,9 * * *$ & $-2,0 * * *$ \\
$\mathrm{H}$ & $-1,8 * * *$ & $-2,8 * * *$ & \\
\hline
\end{tabular}

Für die statistische Signifikanz gelten folgende Werte: * $p$-Wert $<5 \%, * * p$-Wert $<1 \%$, *** $p$-Wert $<0,1 \%$

nehmen weiter ab. Lediglich der Preiseffekt für Energieeffizienzklasse $\mathrm{H}$ steigt nach Einführung der Vorlagepflicht auf $-1,5 \%$ an und ist dann nahezu identisch mit dem Preisabschlag der Klasse G. Die These, dass sich systematisch andere Veränderungseffekte erst mit längerer Zeitverzögerung abzeichnen, kann verworfen werden, da auch bei Betrachtung der Ergebnisse nach weiteren sechs Monaten (November 2015) sich sowohl die Energieeffizienzklassen $\mathrm{A}^{+}$und $\mathrm{A}(6,4 \%$ und 5,7\%) als auch $\mathrm{G}$ und

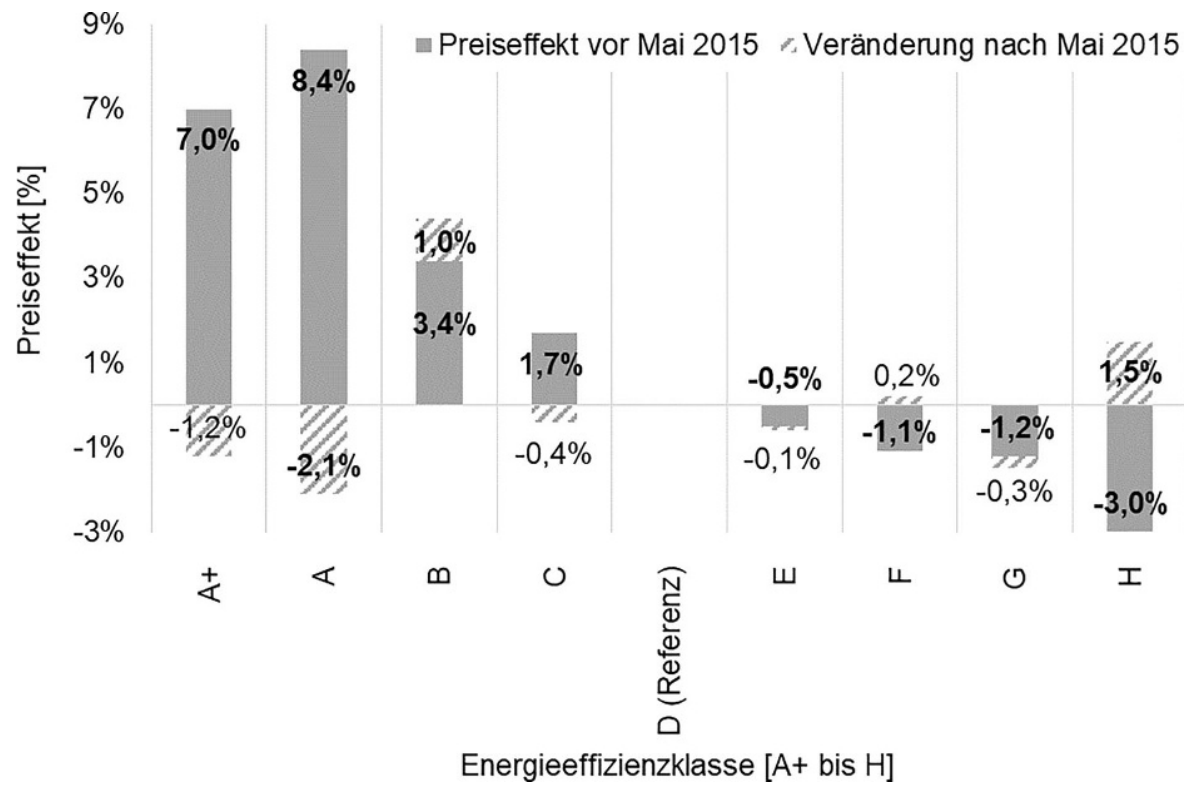

Abb. 4 Veränderung des Mietpreiseffektes des Energieausweises nach Einführung der Vorlagepflicht. Die fett dargestellten Werte weisen eine statistische Signifikanz auf dem 0,1\%-Niveau auf 
H (-1,1\% und $-1,3 \%)$ angleichen. Auch sinken bzw. bleiben bei einer späteren Betrachtung die Preisabschläge für weniger effiziente Wohnungen niedrig.

Sowohl die Immobilienmarktsituation als auch die Einführung der verbindlichen Vorlagepflicht wirken sich auf den Preiseffekt aus. In angespannten Märkten weisen Mieten eine durchschnittlich höhere Sensitivität für die Energieeffizienz auf. Die Intensivierung der Preiseffekte lässt zudem auf eine höhere Markttransparenz durch die Vorlagepflicht schließen, was darauf hindeutet, dass die Anpassung der gesetzlichen Rahmenbedingungen den Energieausweis und dessen Wahrnehmung gestärkt hat.

\section{Fazit und Empfehlungen}

Der Energieausweis wurde eingeführt, um eine erhöhte Markttransparenz zu schaffen sowie ineffizienten Entscheidungen auf dem Immobilienmarkt vorzubeugen. Bei der Umsetzung energiepolitischer Klimaschutzziele ist zudem von Bedeutung, dass eine erhöhte Zahlungsbereitschaft für Energieeffizienz Anreize für energetische Sanierungen und Modernisierungen setzen kann. Das Bewusstsein für Energieeffizienz wird dabei maßgeblich durch den Energieausweis als leicht verfügbares Informationsinstrument geschaffen.

Der Energieausweis wird von den befragten Experten auch nach Einführung der Vorlagepflicht weiterhin kritisch gesehen. Gründe hierfür liegen den Experten zufolge im mangelnden Verständnis seitens der potenziellen Mieter und Käufer, in der defizitären Umsetzung sowie der fragwürdigen Wirtschaftlichkeit einer Investition in energieeffiziente Immobilien. Die Ergebnisse der CBCA belegen jedoch, dass dem Energiekennwert und der Energieeffizienzklasse eine vergleichsweise hohe relative Wichtigkeit bei Mietimmobilien beigemessen wird, wenn die Präferenzen implizit abgefragt werden. Diese experimentelle Analyse zeigt dementsprechend, dass der Einfluss des Informationsinstruments in hypothetischen Auswahlsituationen größer ist, als die Experten vermuten. Mit den wachsenden energetischen Anforderungen an Immobilien durch die EnEV, dem zunehmenden Umweltbewusstsein sowie den steigenden Energiepreisen sind zudem grundsätzlich geeignete Voraussetzungen vorhanden, um den Energieausweis in seiner Bedeutung zu stärken. Zusätzlich sollte der Energieausweis weiterentwickelt und die Marktteilnehmer für seinen Informationswert sensibilisiert werden.

Den Ergebnissen der CBCA entsprechend verdeutlicht das hedonische Preismodell, dass Mieter im realen Immobilienmarkt bereit sind, einen Preisaufschlag für energieeffiziente Wohnungen zu zahlen. Dieser Preiseffekt variiert basierend auf der Immobilienmarktsituation, sodass z. B. in Wohnungsmärkten mit einem Nachfrageüberhang ein höherer Preisaufschlag gezahlt wird. Die Motivation für die Berücksichtigung des energetischen Zustands ist jedoch vielfältig und reicht über den finanziellen Anreiz der Energiekosteneinsparung hinaus. Die Ursachen des nachgewiesenen Preiseffektes bedürfen daher einer weiterführenden Analyse. Gleichzeitig lassen sich die Ergebnisse auf wenig effiziente Wohnungen nur bedingt spiegeln. Da die Preisabschläge für diese Wohnungen gering und über die Effizienzklassen ähnlich sind, scheinen Mieter gegenüber der genauen Ausprägung der Effizienzklasse 
im niedrigeffizienten Bereich nahezu indifferent zu sein. Regulatorische Anpassungen wie die verbindliche Vorlagepflicht können den Energieausweis in seiner Rolle hingegen stärken, insbesondere, wenn über dieses Recht ausreichend informiert und der Ausweis den Interessenten tatsächlich zugänglich gemacht werden muss.

Trotzdem bestehen weiterhin Hemmnisse, die dazu führen, dass der Energieausweis noch nicht seine volle Wirkung zur Erhöhung der Immobilienmarkttransparenz entfalten kann. Ein Grund besteht in Ausnahmeregelungen für die verbindliche Vorlagepflicht, sodass beispielsweise 24\% der Immobilien auch für das Jahr 2016 weiterhin keine Daten zum Energieausweis aufweisen; auch wenn dieser Wert seit der Vorlagepflicht erheblich sank, so betrug er noch im Jahr 2013 89\%. Darüber hinaus könnten Mieter in angespannten Wohnungsmärkten eine Benachteiligung gegenüber Mitbewerbern durch die Einforderung des Energieausweises beim Vermietungsprozess vermuten und daher darauf verzichten. Dies wird dadurch begünstigt, dass ein Verstoß gegen die Vorlagepflicht zwar eine Ordnungswidrigkeit darstellt und mit einer Geldstrafe belegt ist, die Ausweispflicht aber kaum nachgehalten und kontrolliert wird.

Möglichkeiten für die politischen Akteure diesen Hemmnissen entgegenzuwirken setzen sich aus zwei Komponenten zusammen: zum einen die Anpassung des Energieausweises sowie der regulatorischen Rahmenbedingungen, zum anderen eine verstärkte Sensibilisierung der Marktteilnehmer. Zunächst sollte eine Vereinheitlichung des Energieausweises angestrebt werden. Das duale System aus Bedarfs- und Verbrauchsausweis sorgt bislang für eine mangelhafte Vergleichbarkeit der Energieeffizienzkennwerte. Des Weiteren sprechen sich sowohl Experten als auch Immobiliennutzer für die Einführung eines Heizkostenkennwertes aus, der unmittelbar über Kosteneinsparungen aufklärt. Stichprobenartige Kontrollen und die Sanktionierung von Falschangaben stellen weitere Maßnahmen dar. Da jedoch die Zuständigkeiten nicht eindeutig geregelt sind, müssten die rechtlichen Vorgaben erweitert oder konkretisiert werden. Essenziell ist darüber hinaus die Kommunikation des Informationswertes. Ziel beim Umgang mit dem Energieausweis könnte sein, den selbstverständlichen Stellenwert als Entscheidungskriterium zu erreichen, der sich bereits bei der Nutzung des Energielabels von Elektrogeräten eingestellt hat. Dies würde den Energieausweis in seiner Bedeutung für die Erreichung der Klimaschutzziele der Bundesregierung insbesondere auf dem Weg zu einem klimaneutralen Gebäudesektor bis zum Jahr 2050 stärken.

Förderung Dieses Forschungsprojekt wurde durch das Ministerium für Innovation, Wissenschaft und Forschung (MIWF) des Landes Nordrhein-Westfalen im Rahmen des Kompetenzzentrums Verbraucherforschung NRW (KVF NRW) gefördert. Das KVF NRW ist ein Kooperationsprojekt des MIWF mit der Verbraucherzentrale Nordrhein-Westfalen e. V. und dem Ministerium für Klimaschutz, Umwelt, Landwirtschaft, Natur- und Verbraucherschutz (MKULNV) des Landes Nordrhein-Westfalen.

Open Access Dieser Artikel wird unter der Creative Commons Namensnennung 4.0 International Lizenz (http://creativecommons.org/licenses/by/4.0/deed.de) veröffentlicht, welche die Nutzung, Vervielfältigung, Bearbeitung, Verbreitung und Wiedergabe in jeglichem Medium und Format erlaubt, sofern Sie den/die ursprünglichen Autor(en) und die Quelle ordnungsgemäß nennen, einen Link zur Creative Commons Lizenz beifügen und angeben, ob Änderungen vorgenommen wurden. 
Danksagung Für die Daten- und Softwarebereitstellung bedanken wir uns bei der Immobilien Scout $\mathrm{GmbH}$, GfK Geomarketing GmbH, Verivox GmbH, Sawtooth Software Inc. und ESRI Deutschland $\mathrm{GmbH}$

\section{Literatur}

Adjei A, Hamilton L, Roys M (2011) A study of homeowners' energy efficiency improvements and the impact of the Energy Performance Certificate. BRE (Building Research Establishment), Hertfordshire

Amecke H (2012) The impact of energy performance certificates: a survey of German home owners. Energy Policy 46:4-14

Banfi S, Farsi M, Filippini M, Jakob M (2008) Willingness to pay for energy-saving measures in residential buildings. Energy Econ 30:503-516

Brounen D, Kok N, Quigley JM (2013) Energy literacy, awareness, and conservation behavior of residential households. Energy Econ 38:42-50

Bundesministerium für Umwelt, Naturschutz, Bau und Reaktorsicherheit (BMUB) (Hrsg) (2016) Wege zum Effizienzhaus Plus: Grundlagen und Beispiele für energieerzeugende Gebäude. https:// www.bmi.bund.de/SharedDocs/downloads/DE/publikationen/themen/bauen/energieeffizienzhausplus.pdf?_blob=publicationFile\&v=1. Zugegriffen: 9. Okt. 2018

Bundesministerium für Umwelt, Naturschutz, Bau und Reaktorsicherheit (BMUB) (Hrsg) (2017) Umweltbewusstsein in Deutschland 2016. Ergebnisse einer repräsentativen Bevölkerungsumfrage. https://www.umweltbundesamt.de/sites/default/files/medien/376/publikationen/umweltbewusstsein deutschland_2016_bf.pdf. Zugegriffen: 9. Okt. 2018

Bundesgesetzblatt (BGBl) (Hrsg) (2007) Verordnung über energiesparenden Wärmeschutz und energiesparende Anlagentechnik bei Gebäuden (Energieeinsparverordnung - EnEV) Teil I Nr. 34:1519-1563

Chau CK, Tse MS, Chung KY (2010) A choice experiment to estimate the effect of green experience on preferences and willingness-to-pay for green building attributes. Build Environ 45:2553-2561

Christensen TH, Gram-Hanssen K, de Best-Waldhober M, Adjei A (2014) Energy retrofits of Danish homes: is the Energy Performance Certificate useful? Build Res Inf 42:489-500

Conway D, Li CQ, Wolch J, Kahle C, Jerrett M (2010) A spatial autocorrelation approach for examining the effects of urban greenspace on residential property values. J Real Estate Finance Econ 41:150-169

Deschermeier P, Haas H, Voigtländer M (2015) Anbieter von günstigem Wohnraum - eine Analyse für zehn Großstädte in NRW: In Kooperation mit der LEG Immobilien AG. IW policy paper 35

Ebert T, EBig N, Hauser G (2010) Zertifizierungssysteme für Gebäude: Nachhaltigkeit bewerten - Internationaler Systemvergleich - Zertifizierung und Ökonomie. De Gruyter, Detail, Berlin, München

Eichholtz P, Kok N, Quigley JM (2010) Doing well by doing good? Green office buildings. Am Econ Rev 100:2492-2509

Fuerst F, McAllister P, Nanda A, Wyatt P (2015) Does energy efficiency matter to home-buyers? An investigation of EPC ratings and transaction prices in England. Energy Econ 48:145-156

Glück S (1997) Immobilienmakler und Neue Institutionenökonomik. Deutscher Universitätsverlag, Wiesbaden

Green PE, Srinivasan V (1978) Conjoint analysis in consumer research: issues and outlook. J Consum Res 5:103-123

Green PE, Srinivasan V (1990) Conjoint analysis in marketing: new developments with implications for research and practice. J Mark 54:3-19

Henger R, Voigtländer M (2014) Transaktions- und Angebotsdaten von Wohnimmobilien: Eine Analyse für Hamburg. IW Trends Vierteljahresschr Wirtschaftsforsch 41:85-100

Henger R, Schier M, Voigtländer M (2015) Der künftige Bedarf an Wohnungen: Eine Analyse für Deutschland und alle 402 Kreise. IW policy paper 24

Hyland M, Lyons R, Lyons S (2013) The value of domestic building energy efficiency - evidence from Ireland. Energy Econ 40:943-952

Information und Technik Nordrhein-Westfalen, Geschäftsbereich Statistik (Hrsg) (2018) Wohnungsbestand in den Gemeinden Nordrhein-Westfalens am 31. Dezember 2017. https://webshop.it.nrw.de/ gratis/F249\%20201700.pdf. Zugegriffen: 4. Okt. 2018

IPCC (2014) Climate change 2014: mitigation of climate change: working group III contribution to the fifth assessment report of the intergovernmental panel on climate change. Cambridge University Press, Cambridge 
Kahn ME, Kok N (2014) The capitalization of green labels in the California housing market. Reg Sci Urban Econ 47:25-34

Kaiser I, Neukirchner J, Bodendorf F (2011) Energieeffizientes Bauen - IT-gestützte Marktkenntnis und Markterkenntnis. Wirtschaftsinform Manag 3:46-53

Kok N, Jennen M (2012) The impact of energy labels and accessibility on office rents. Energy Policy 46:489-497

Landtag Nordrhein-Westfalen (Hrsg) (2016) Drucksache 16/12353. https://www.landtag.nrw.de/portal/ WWW/dokumentenarchiv/Dokument?Id=MMD16\%2F12353\%7C1\%7C0. Zugegriffen: 4. Okt. 2018

LEG Immobilien AG (Hrsg) (2018) LEG-Wohnungsmarktreport NRW 2018. https://www.leg-wohnen.de/ fileadmin/user_upload/Assets/PDFs/Unternehmen/Presse/

Wohnungsmarktreporte/Wohnungsmarktreport_2018/LEG-Wohnungsmarktreport_NRW_2018.pdf. Zugegriffen: 2. Okt. 2018

Lützkendorf T, Lorenz D (2007) „Green Buildings“ - nur umweltfreundlich oder auch wirtschaftlich und wertstabil. In: Verband Deutscher Pfandbriefbanken (Hrsg) Immobilien-Banking, 6. Aufl., S 58-68

Mansour OE, Radford SK (2016) Rethinking the environmental and experiential categories of sustainable building design: a conjoint analysis. Build Environ 98:47-54

McCullough D (2002) A user's guide to conjoint analysis. Mark Res 14:18-23

an de Meulen P, Micheli M, Schaffner S (2014) Documentation of German real estate market data - sample of real estate advertisements on the internet platform ImmobilienScout24 2007-2013. RWI Materialien 80

Mieterschutzverein für Aachen und Umgegend e. V., Aachener Haus \& Grundbesitzer-Verein e. V., Initiative Aachen e. V. (Hrsg) (2016) Mietspiegel für das Stadtgebiet Aachen 2016. http://www.aachen.de/ BIS/FO/1060Mietspiegel2016.pdf. Zugegriffen: 9. Okt. 2018

Murphy L (2014) The influence of the Energy Performance Certificate: the Dutch case. Energy Policy 67:664-672

Nier H (2018) Diese Wohnung suchen die Deutschen. https://de.statista.com/infografik/15557/ meistgesuchte-wohnungen-deutschlands. Zugegriffen: 2. Okt. 2018

Orme B (2000) Hierarchical Bayes: Why all the attention? Research paper series. Sawtooth Software, Sequim

Orme B (2002) Formulating attributes and levels in conjoint analysis. Research paper series. Sawtooth Software, Sequim

Rosen S (1974) Hedonic prices and implicit markets: product differentiation in pure competition. J Polit Econ 82:34-55

Sammer K, Wüstenhagen R (2006) The influence of eco-labelling on consumer behaviour - results of a discrete choice analysis for washing machines. Bus Strategy Environ 15:185-199

Sattler H, Hensel-Börner S (2001) A comparison of conjoint measurement with self-explicated approaches. In: Gustafsson A, Herrmann A, Huber F (Hrsg) Conjoint measurement: methods and applications. Springer, Berlin, Heidelberg, S 121-133

Schafhausen F (2013) Die Energiewende - Aufbruch in die Zukunft. Vierteljahresh Wirtschaftsforsch $82: 11-28$

Schober C (2015) Wie können Wirkungen monetarisiert werden? In: Schober C, Then V (Hrsg) Praxishandbuch social return on investment: Wirkung sozialer Investitionen messen. Schäffer-Poeschel, Stuttgart, S 125-160

Statistisches Bundesamt (Hrsg) (2017) Statistisches Jahrbuch 2017: Deutschland und Internationales. Destatis, Wiesbaden

Ürge-Vorsatz D, Novikova A (2008) Potentials and costs of carbon dioxide mitigation in the world's buildings. Energy Policy 36:642-661

Verbraucherzentrale NRW (Hrsg) (2018) Energieausweis: Was sagt dieser Steckbrief für Wohngebäude aus? https://www.verbraucherzentrale.de/wissen/energie/energetische-sanierung/energieausweiswas-sagt-dieser-steckbrief-fuer-wohngebaeude-aus-24074\#bedarfsoderverbrauchsausweis. Zugegriffen: 9. Nov. 2018

Watts C, Jentsch MF, James PA (2011) Evaluation of domestic energy performance certificates in use. Build Serv Eng Res Technol 32:361-376

Weglage A (2010) Praktische Erstellung des Energieausweises. In: Weglage A (Hrsg) Energieausweis Das große Kompendium, 3. Aufl. Vieweg+Teubner, Wiesbaden, S 33-74

Zalejska-Jonsson A (2013) Impact of energy and environmental factors in the decision to purchase or rent an apartment: the case of Sweden. J Sustain Real Estate 5:66-85 